Sharif University of Technology
Scientia Iranica
SCIENTIA

\title{
Vibration control of tapered magnetostrictive plate considering shear correction factor
}

\author{
A. Ghorbanpour Arani*, Z. Khoddami Maraghi and H. Khani Arani \\ Department of Solid Mechanics, Faculty of Mechanical Engineering, University of Kashan, Kashan, Iran.
}

Received 14 September 2014; received in revised form 12 June 2015; accepted 25 August 2015

\author{
KEYWORDS \\ Free vibration; \\ Magnetostrictive \\ rectangular plate; \\ Taper ratio; \\ Feedback control \\ system; \\ Differential \\ quadrature method.
}

\begin{abstract}
In this research, dynamic response of tapered plate made of MsM (magnetostrictive material) is studied for the first time. First-order Shear Deformation Theory (FSDT) is used to derive the governing equations of tapered MsP (magnetostrictive plate) while the thickness varies linearly. To enhance accuracy of the results, shear correction factor is considered and a feedback control system is utilized to investigate the effects of magnetic field on MsP. The five equations of motion that are obtained by Hamilton's principle are solved using Differential Quadrature Method (DQM) and compared with those available in the literature. Results indicate the effect of various parameters such as aspect ratio, thickness ratio, taper ratio, boundary conditions, and the controller effect of velocity feedback gain on the frequency of MsP. These findings can be used for active noise and vibration cancellation systems in many smart structures.
\end{abstract}

(C) 2016 Sharif University of Technology. All rights reserved.

\section{Introduction}

Magnetostriction in MsM, such as iron, Terfenol-D, Ferrite, Nickel, Cobalt, and their alloys, is a phenomenon only found in ferromagnetic materials. The magnetostrictive principle is based on certain magnetomechanical properties of these materials. MsMs deform when exposed to magnetic field. The magnetostrictive effect is an interaction of magnetic and mechanical parameters of ferromagnetic materials. These materials have applications in development of electrical machinery, fusion reactors, communications equipment, and computers [1-3]. The literature contains some papers that have analyzed MsM and then reviewed previous works about plates with variable thickness.

\footnotetext{
*. Corresponding author. Tel.: +983155912450; Fax: + 983155912424 E-mail address: aghorban@kashanu.ac.ir (A. Ghorbanpour Arani)
}

Moon and Pao [4] studied the instability of a beam-plate in a transverse magnetic field. They presented a mathematical model according to distributed magnetic torques along the plate. Their results indicated that buckling may occur when the uniform magnetic field intensity reaches a critical value. The dynamic stability of ferromagnetic rectangular plate under transverse magnetic field and in-plane periodic compression for simply supported boundary condition was presented by Wang and Lee [5]. They considered the effects of magnetic damping and excitation frequency and solved the equation of motion of ferromagnetic plate by means of a linearized magnetoelastic theory and perturbation technique. Moita et al. [6] investigated a higher-order Finite Element Model (FEM) for static and free vibration analyses of magneto-electro-elastic thin and thick plates. Solutions were obtained for different laminations of the magnetoelectro-elastic and purely elastic plates. Free vibration of Functionally Graded (FG) thick rectangular plates was presented by Hashemi et al. [7] for two opposite 
edges simply supported and all possible combinations of free, simply supported, and clamped boundary conditions. Their plate was embedded in Winkler or Pasternak elastic foundations. They derived and exactly solved the equations of motion using FSDT of plate by considering the shear correction factors. Hong [8] proposed thermal sinusoidal vibration and transient response of magnetostrictive FG material plates without shear correction factor by using the generalized DQM. Also, Hong [9] presented, in another work, thermal vibration and transient response of Terfenol-D FG material plates by using FSDT theory. He used the generalized DQM for simply supported boundary conditions for the center displacement. He also analyzed shear correction coefficient values, thickness of mounted magnetostrictive layer, control gain values, temperature of environment, and different boundary conditions.

Plates with variable thicknesses are used in aerospace, ocean structures, turbine disk, and mechanical, civil, and electronics engineering. These plates have applications in altering resonant frequency and reducing the size and weight of the structure. Therefore, it is very important to understand free vibrations of plates with variable thicknesses $[10,11]$.

Kukreti and Fursa [12] studied the fundamental frequency of simply supported, thin rectangular plates with a linear taper in one direction. They used Ritz and DQM to obtain the displacements and solved the free vibration governing differential equation of motion. They also compared their results with those of the finite element method. Free vibration of isotropic and orthotropic rectangular plates was presented by Civalek [10] with linearly varying thicknesses along one direction and for different types of boundary conditions. Thermal gradient effect on vibration of non-homogeneous orthotropic plate with linearly varying thickness was proposed by Gupta and Sharma [13] for clamped-simply Supported-ClampedSimply Supported (CSCS). They considered the effect of taper constant and aspect ratio on first and second modes of vibration. Kang and Kim [14] investigated free vibration of a simply supported rectangular plate with unidirectional arbitrary-thickness variation. They reported the results for linearly varying and quadratic thickness function and mode shapes compared with other analytical methods including FEM (ANSYS). Singh and Saxena [15] presented transverse vibration of a rectangular plate with bidirectional thickness variation for different combinations of boundary conditions at the four edges. They obtained the first three frequencies with positive and negative taper parameters. The mode shapes were plotted for several cases in this work. Effect of linear thickness variations in both directions on vibration of viscoelastic rectangular plate was studied by Gupta and Khanna [16]. They derived the equations of motion by using RayleighRitz technique with a two-term deflection function for clamped boundary conditions on all the four edges.

In addition to the use of FSDT in this research, the advanced Higher order Shear Deformation Theories (HSDTs) are also introduced in this section to consider the thickness stretching effect and the transverse shear deformation effects without shear correction factors. Since the displacement field of advanced HSDTs is the function of thickness of plate, sophisticated mathematical calculations are required to compute total energy of the system. In this regard, some of the advanced HSDTs have been provided in this section for researchers who would like to continue this work at a higher level.

Tounsi et al. [17] presented a refined trigonometric shear deformation theory for thermo-elastic bending of FG sandwich plates. The number of unknown functions involved was only four. This theory did not require shear correction factor. They expressed the displacement components by trigonometric series through the thickness of plate in order to develop a twodimensional theory. Shear stress free surface conditions are satisfied in this theory because the transverse shear stresses vary parabolically across the thickness of plate. This paper investigated the influences of transverse shear deformation, thermal load, plate aspect ratio, and volume fraction distribution.

An efficient and simple higher order shear and normal deformation theory for FG plates was analyzed by Belabed et al. [18]. In this theory, the transverse displacement is divided into the bending, shear, and thickness stretching parts. Thus, the number of unknowns for the present theory is reduced to five, as opposed to six or even greater numbers in the case of other shear and normal deformation theories. Considering hyperbolic variation of all displacements across the thickness, both shear deformation and thickness stretching effects are applied in this theory. Also, the present theory accounts the stress-free boundary conditions on the upper and lower surfaces of the plate without requiring any shear correction factor. Hebali et al. [19] developed a new quasi-three-dimensional (3D) hyperbolic shear deformation theory for the bending and free vibration analysis of FG plates. Like the previous paper, the transverse displacement in this theory is divided into three parts including bending, shear, and thickness stretching parts. Their theory satisfies the zero traction boundary conditions on the surfaces of the plate without using shear correction factor and accounts for both transverse shear and normal deformations. They concluded that the inclusion of thickness stretching effect made a plate stiffer and hence led to a reduction in deflection and an increase in frequency. 
Bending of FG material plate resting on elastic foundation was studied by Zidi et al. [20]. The plate was subjected to hygro-thermo-mechanical loading and was analyzed using a four-variable refined plate theory. This theory satisfies both quadratic variation of the transverse shear strains across the thickness and the zero traction boundary conditions on the top and bottom surfaces of the plate without shear correction factors. Also, the number of unknowns in the present theory is only four. More details of these theories have been presented in [21-24].

Despite the mentioned papers, the tapered $\mathrm{MsPs}$ are analyzed for the first time in this work, where thickness of $\mathrm{MsP}$ is varied linearly in $x$ direction. MsM, due to its reciprocal nature, is one of the most affordable options in control systems. Magnetomechanical coupling in MsM, like electro-mechanical coupling in piezoelectric materials, can be used in stability of systems. Using a feedback control system, the magnetic field is varied in order to study the vibrational behavior of MsP. In this work, the variation in magnetization of plate is investigated by considering different aspect ratios, thickness ratios, taper ratios, and boundary conditions.

Variable thickness plates are commonly used in aeronautical, mechanical, and ocean structures in order to reduce the weight of structures and to improve their dynamical behaviors. Plates of various geometries are also key components in many structural and machinery applications, particularly in aerospace, civil, and automotive industries. Since the variable thickness plates change the resonant frequency, free vibration analysis of such plates is essential to have an efficient and reliable design $[25,26]$.

The result of this study can be useful to reduce the damage caused by the destructive vibrations and control the systems such as electro-hydraulic actuator, wireless linear motors, etc.

\section{Tapered plate}

A rectangular plate with the plane dimensions of $(a \times b)$ is considered, as shown in Figure 1. The thickness of plate varies linearly in the $x$ direction based on $h(x)=$ $h_{0}\left(1+\beta \frac{x}{a}\right)$ according to Figure 1 . Where $\beta$ is named taper ratio and $\beta=0$ means the thickness of plate is constant. Also, in this work, the taper ratio changes between $-1<\beta<1$.

\section{Magneto-mechanical coupling in MsM}

MsMs rely on the magnetostrictive effect where the deformation is induced along the applied external magnetic field $[1,2]$.

The stress-strain relation for isotropic MsM $[27,28]$, and the magneto-mechanical coupling in
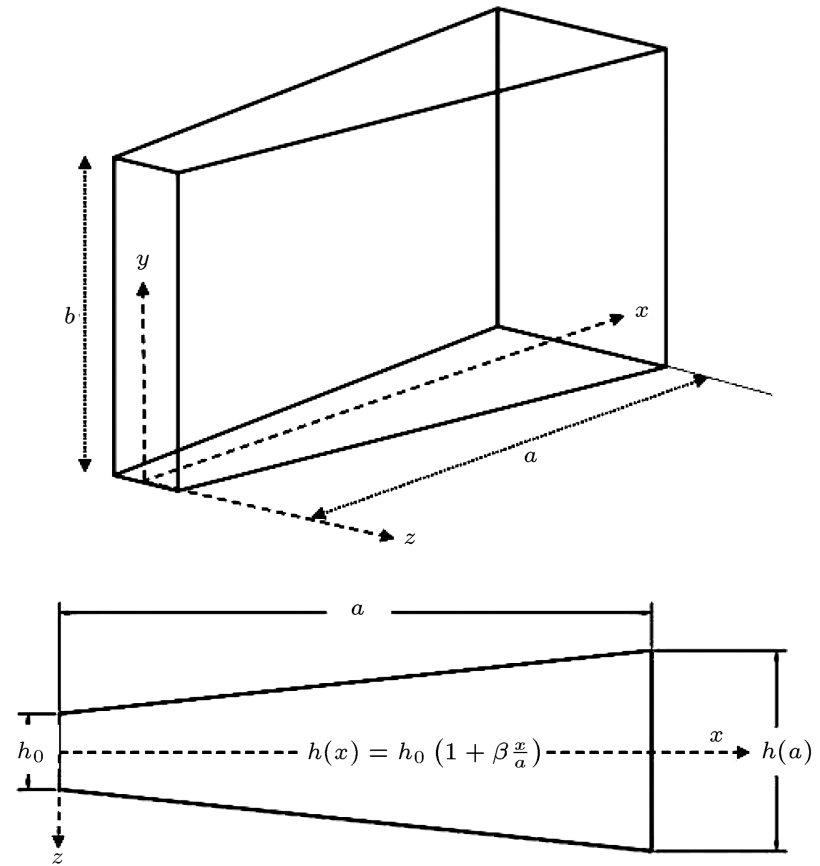

Figure 1. Geometry and coordinate of tapered plate.

these materials can be observed in Eq. (1):

$$
\begin{aligned}
{\left[\begin{array}{c}
\sigma_{x x} \\
\sigma_{y y} \\
\sigma_{z z} \\
\sigma_{x y} \\
\sigma_{x z} \\
\sigma_{y z}
\end{array}\right]=} & {\left[\begin{array}{cccccc}
\bar{Q}_{11} & \bar{Q}_{12} & 0 & 0 & 0 & 0 \\
\bar{Q}_{21} & \bar{Q}_{22} & 0 & 0 & 0 & 0 \\
0 & 0 & 0 & 0 & 0 & 0 \\
0 & 0 & 0 & \bar{Q}_{44} & 0 & 0 \\
0 & 0 & 0 & 0 & \bar{Q}_{55} & 0 \\
0 & 0 & 0 & 0 & 0 & \bar{Q}_{66}
\end{array}\right]\left[\begin{array}{c}
\varepsilon_{x x} \\
\varepsilon_{y y} \\
\varepsilon_{z z} \\
2 \varepsilon_{x y} \\
2 \varepsilon_{x z} \\
2 \varepsilon_{y z}
\end{array}\right] } \\
& -\left[\begin{array}{lll}
0 & 0 & e_{31} \\
0 & 0 & e_{32} \\
0 & 0 & e_{34}
\end{array}\right]\left[\begin{array}{c}
0 \\
0 \\
H_{z}
\end{array}\right]
\end{aligned}
$$

where $\sigma_{i j}$ and $\varepsilon_{i j}$ are stress and strain, respectively. As seen from the constitutive model, the induced strain is always positive and independent of the direction of the applied magnetic field. Also $Q_{i j}$ are the terms of engineering constants:

$$
\begin{aligned}
& \bar{Q}_{11}=\bar{Q}_{22}=\frac{E}{\left(1-v^{2}\right)}, \\
& \bar{Q}_{12}=\frac{E v}{\left(1-v^{2}\right)}, \\
& \bar{Q}_{66}=\bar{Q}_{55}=k_{f} \frac{E}{2(1+v)}, \\
& \bar{Q}_{44}=\frac{E}{2(1+v)},
\end{aligned}
$$

in which $E, v$, and $k_{f}$ are Young modulus, Poisson's ratio, and shear correction factor, respectively. It is necessary to consider the shear correction factor to 
compute transverse shear forces. The shear correction factor is typically taken to be $5 / 6$ for homogeneous plates. Also, it may be a function of material properties and the geometric dimension of plate [7]. In this regard, Timoshenko [29] presented the following relation:

$$
k_{f}=\frac{5+5 v}{6+5 v} \text {. }
$$

MsMs possess magnetic anisotropy in their atomic structure and, therefore, they undergo dimensional changes when placed in magnetic fields as a result of reorientation (of the atomic magnetic moments) [30]. Similar to electrostrictive materials, the one-dimensional constitutive model for converse effect in magnetostrictive materials can be represented as $\sigma_{i}=C_{i k} \varepsilon_{k}+e_{k i} H_{k}$, where $C_{i k}, k, \varepsilon_{k}$, and $H$ are stress, stiffness coefficient, strain, magnetostrictive constant, and magnetic field, respectively [31]. Magnetic induction is introduced as $B_{i}=e_{i k} \varepsilon_{k}+\mu_{k i} H_{k}$, in which $\mu_{k i}$ is magnetic permeability coefficient. Also, $H_{k}$ is equal to $\frac{\partial \psi_{k}}{\partial x_{k}}$, where $\psi_{k}$ is the magnetic potential [32]. Hence, the most common configurations for these actuators are rod or cylindrical element wrapped in an exciting coil.

In this research, another model is used to follow the magnetization effect on $\mathrm{MsP}$ where a feedback control system investigates the intensity of magnetic field. Chen et al. [31] and Pan et al. [32] have focused on magnetic potential.

$e_{i j}$ are magnetostrictive coupling modules, which are determined as follow [27]:

$$
\begin{aligned}
& e_{31}=\tilde{e}_{31} \cos ^{2} \theta+\tilde{e}_{32} \sin ^{2} \theta \\
& e_{32}=\tilde{e}_{31} \sin ^{2} \theta+\tilde{e}_{32} \cos ^{2} \theta \\
& e_{34}=\left(\tilde{e}_{31}-e_{32}\right) \sin \theta \sin \theta,
\end{aligned}
$$

where $\theta$ represents the direction along which a given magnetic anisotropy may have been induced. In this study, Terfenol-D is used as a magnetostrictive alloy that is sensitive to changes in magnetic field. A unidirectional magnetic field is applied on Terfenol-D in order to follow the magnetization effect on $\mathrm{MsP}$ in a feedback control system. Since only $e_{31}$ and $e_{32}$ and $e_{34}$ have been presented for Terfenol-D, the magnetic field is considered in $z$ direction.

$H_{z}$ is the magnetic field intensity and can be expressed as follows $[27,33,34]$ :

$$
H_{z}=K_{c} I(x, y, t)=K_{c} C(t) \frac{\partial \tilde{w}(x, y, z, t)}{\partial t},
$$

where $K_{c}=n_{c} / \sqrt{b_{c}^{2}+4 r_{c}^{2}}$, in which $b_{c}$ is the coil width, $r_{c}$ is coil radius, and $n_{c}$ is number of turns in the coil. $I(t), \tilde{W}(x, y, z, t)$, and $C(t)$ are the coil current, transvers deflection of $\mathrm{MsP}$, and the control gain, respectively, which is assumed to be constant in this study.

\section{Energy method to derive motion equations}

The energy method is used to obtain the governing equation in this work. In this regard, the strain energy and kinetic energy of an elastic body, such as rectangular plate, is expressed as [35]:

$$
\begin{aligned}
U= & \frac{1}{2} \int_{-h(x) / 2}^{h(x) / 2} \int_{0}^{b} \int_{0}^{a}\left(\sigma_{x x} \varepsilon_{x x}+\sigma_{y y} \varepsilon_{y y}+\tau_{x y} \gamma_{x y}\right. \\
& \left.+\tau_{x z} \gamma_{x z}+\tau_{y z} \gamma_{y z}\right) d x d y d z,
\end{aligned}
$$

where $\sigma$ and $\varepsilon$ are normal stress and strain and $\tau$ and $\gamma$ are shear stress and strain of plate. Also, the kinetic energy of the MsP is [35]:

$$
K=\frac{\rho_{m} A}{2} \int_{0}^{b} \int_{0}^{a}\left[\left(\frac{\partial \tilde{U}}{\partial t}\right)^{2}+\left(\frac{\partial \tilde{V}}{\partial t}\right)^{2}+\left(\frac{\partial \tilde{W}}{\partial t}\right)^{2}\right] d x d y
$$

where $\rho_{m}$ and $A$ are the mass density and cross section area of $\mathrm{MsP}$.

\subsection{First-order Shear Deformation Theory (FSDT)}

The Mindlin-Reissner theory of plates is an extension of Kirchhoff-Love plate theory and it is often called the FSDT with the following assumptions [36]:

- Shear deformations through-the-thickness of a plate are taken into account;

- Normal to the mid-surface remains straight but not necessarily perpendicular to the mid-surface;

- FSDT is used to calculate the deformations and stresses in a plate whose thickness is one-tenth the planar dimensions while the Kirchhoff-Love theory is applicable to thinner plates;

- FSDT includes in-plane shear strains while Kirchhoff-Love plate theory incorporates first-order shear effects.

HSDT is based on the same assumptions as those of the Classical Plate Theory (CPT) and FSDT, except that the assumption of the straightness and normality of the transverse normal is relaxed [37]. It is worth to mention that FSDT requires shear correction factor and neglects the stretching effect while HSDTs account for the transverse shear deformation effects without requiring shear correction factors [38]. As mentioned in the introduction section, since the displacement field of advanced HSDTs is the function of thickness of plate, sophisticated mathematical calculations are required to compute total energy of the system. Therefore, FSDT is used in this study.

According to the FSDT of plate, the displacement 
components of the middle surface along the $x, y$, and $z$ axes, shown by $\tilde{U}, \tilde{V}$ and $\tilde{W}$, can be expressed as:

$$
\begin{aligned}
& \tilde{U}(x, y, z, t)=u_{0}(x, y, t)+z \theta_{1}(x, y, t), \\
& \tilde{V}(x, y, z, t)=v_{0}(x, y, t)+z \theta_{2}(x, y, t), \\
& \tilde{W}(x, y, z, t)=w_{0}(x, y, t),
\end{aligned}
$$

where $u_{0}(x, y, t), v_{0}(x, y, t)$, and $w_{0}(x, y, t)$ are displacement along $(x, y, z)$ direction and $\theta_{1}(x, y, t)$, and $\theta_{2}(x, y, t)$ are rotations about $x$ and $y$ axes.

According to Hooke's law, the linear strain relations are presented by:

$$
\begin{aligned}
\varepsilon_{x x}= & \frac{\partial}{\partial x} u_{0}(x, y, t)+z\left(\frac{\partial}{\partial x} \theta_{1}(x, y, t)\right), \\
\varepsilon_{y y}= & \frac{\partial}{\partial y} v_{0}(x, y, t)+z\left(\frac{\partial}{\partial y} \theta_{2}(x, y, t)\right), \\
\varepsilon_{z z}= & 0, \\
\varepsilon_{x y}= & \frac{1}{2} \frac{\partial}{\partial x} v_{0}(x, y, t)+\frac{1}{2} z\left(\frac{\partial}{\partial x} \theta_{2}(x, y, t)\right) \\
& +\frac{1}{2} \frac{\partial}{\partial y} u_{0}(x, y, t)+\frac{1}{2} z\left(\frac{\partial}{\partial y} \theta_{1}(x, y, t)\right), \\
\varepsilon_{x z}= & \frac{1}{2} \frac{\partial}{\partial x} w_{0}(x, y, t)+\frac{1}{2} \theta_{1}(x, y, t), \\
\varepsilon_{y z}= & \frac{1}{2} \frac{\partial}{\partial y} w_{0}(x, y, t)+\frac{1}{2} \theta_{2}(x, y, t) .
\end{aligned}
$$

\subsection{Hamilton's principle}

According to Hamilton's principle, the first variation form of motion equations must be zero [35]:

$$
\delta \int_{t_{1}}^{t_{2}}[K-U] d t=0 .
$$

Substituting Eqs. (6) and (7) in Eq. (10) and considering following dimensionless parameters:

$$
\begin{aligned}
& (\zeta, \eta)=\left(\frac{x}{a}, \frac{y}{b}\right), \quad(U, V, W)=\left(\frac{u_{0}}{a}, \frac{v_{0}}{b}, \frac{w_{0}}{h_{0}}\right), \\
& \Theta_{1}=\theta_{1}, \quad \Theta_{2}=\theta_{2}, \quad(\alpha, \mu, \gamma)=\left(\frac{h_{0}}{a}, \frac{h_{0}}{b}, \frac{a}{b}\right), \\
& G_{i j}=\frac{e_{i j} C(t) K_{c}}{\sqrt{E \rho_{m}}}, \quad Q_{i j}=\frac{\bar{Q}_{i j}}{E}, \quad \tau=\frac{t}{a} \sqrt{\frac{E}{\rho_{m}}} .
\end{aligned}
$$

The equations of motion are obtained by setting the coefficients $\delta U, \delta V, \delta W, \delta \theta_{1}$, and $\delta \theta_{2}$ equal to zero as follows:

$$
\begin{aligned}
& \delta U: Q_{11} \alpha((\zeta) \beta+1) \frac{d^{2} U}{d \zeta^{2}}+\frac{1}{2} Q_{66} \mu \gamma((\zeta) \beta+1) \frac{d^{2} U}{d \eta^{2}} \\
& +Q_{11} \alpha \beta \frac{d U}{d \zeta}+\frac{1}{2} Q_{21} \alpha \beta \frac{d V}{d \eta} \\
& +\frac{1}{2} Q_{21} \alpha((\zeta) \beta+1) \frac{d^{2} V}{d \eta d \zeta}+\frac{1}{2} Q_{12} \alpha \beta \frac{d V}{d \eta} \\
& +\frac{1}{2} Q_{12} \alpha((\zeta) \beta+1) \frac{d^{2} V}{d \eta d \zeta} \\
& +\frac{1}{2} Q_{66} \alpha((\zeta) \beta+1) \frac{d^{2} V}{d \eta d \zeta}-\alpha((\zeta) \beta+1) \frac{d^{2} U}{d \tau^{2}} \\
& -\frac{1}{2} G_{31}\left(\alpha^{2} \beta\left(\frac{d W}{d \tau}\right)+\alpha^{2}((\zeta) \beta+1) \frac{d^{2} W}{d \tau d \zeta}\right)=0, \\
& \delta V:+\frac{1}{2} Q_{21} \mu((\zeta) \beta+1) \frac{d^{2} U}{d \eta d \zeta} \\
& +\frac{1}{2} Q_{12} \mu((\zeta) \beta+1) \frac{d^{2} U}{d \eta d \zeta}+\frac{1}{2} Q_{66} \alpha \beta \gamma \frac{d U}{d \eta} \\
& +\frac{1}{2} Q_{66} \mu((\zeta) \beta+1) \frac{d^{2} U}{d \eta d \zeta}+Q_{22} \mu((\zeta) \beta+1) \frac{d^{2} V}{d \eta^{2}} \\
& +\frac{1}{2 \gamma} Q_{66} \alpha \beta \frac{d V}{d \zeta}+\frac{1}{2} \frac{Q_{66} \alpha((\zeta) \beta+1)}{\gamma} \frac{d^{2} V}{d \zeta^{2}} \\
& -\frac{\alpha((\zeta) \beta+1)}{\gamma} \frac{d^{2} V}{d \tau^{2}}-\frac{1}{2} G_{32} \alpha \mu \frac{d^{2} W}{d \eta d \tau}=0, \\
& \delta W:+\frac{1}{2} Q_{44} \mu^{2}((\zeta) \beta+1) \frac{d^{2} W}{d \eta^{2}}+\frac{1}{2} Q_{55} \alpha^{2} \beta \frac{d W}{d \zeta} \\
& +\frac{1}{2} Q_{55} \alpha^{2}((\zeta) \beta+1) \frac{d^{2} W}{d \zeta^{2}}+\frac{1}{2} Q_{55} \alpha \beta \Theta_{1} \\
& +\frac{1}{2} Q_{55} \alpha((\zeta) \beta+1) \frac{d \Theta_{1}}{d \zeta} \\
& +\frac{1}{2} Q_{44} \mu((\zeta) \beta+1) \frac{d \Theta_{2}}{d \eta}-\alpha^{2}((\zeta) \beta+1) \frac{d^{2} W}{d \tau^{2}} \\
& -\frac{1}{2} G_{31} \alpha((\zeta) \beta+1) \frac{d^{2} U}{d \zeta d \tau} \\
& -\frac{1}{2} G_{32} \alpha((\zeta) \beta+1) \frac{d^{2} V}{d \eta d \tau}=0, \\
& \delta \theta_{1}:-\frac{1}{2} Q_{55} \alpha((\zeta) \beta+1) \frac{d W}{d \zeta}-\frac{1}{2} Q_{55}((\zeta) \beta+1) \Theta_{1} \\
& +\frac{1}{24} Q_{66} \mu^{2}((\zeta) \beta+1)^{3} \frac{d^{2} \Theta_{1}}{d \eta^{2}}
\end{aligned}
$$




$$
\begin{aligned}
& +\frac{1}{4} Q_{11} \alpha^{2}((\zeta) \beta+1)^{2} \beta \frac{d \Theta_{1}}{d \zeta} \\
& +\frac{1}{12} Q_{11} \alpha^{2}((\zeta) \beta+1)^{3} \frac{d^{2} \Theta_{1}}{d \zeta^{2}} \\
& +\frac{1}{8} Q_{21} \alpha \mu((\zeta) \beta+1)^{2} \beta \frac{d \Theta_{2}}{d \eta} \\
& +\frac{1}{24} Q_{21} \alpha \mu((\zeta) \beta+1)^{3} \frac{d^{2} \Theta_{2}}{d \eta d \zeta} \\
& +\frac{1}{8} Q_{12} \alpha \mu((\zeta) \beta+1)^{2} \beta \frac{d \Theta_{2}}{d \eta} \\
& +\frac{1}{24} Q_{12} \alpha \mu((\zeta) \beta+1)^{3} \frac{d^{2} \Theta_{2}}{d \eta d \zeta} \\
& +\frac{1}{24} Q_{66} \mu \alpha((\zeta) \beta+1)^{3} \frac{d^{2} \Theta_{2}}{d \eta d \zeta} \\
& -\frac{1}{12} \alpha^{2}((\zeta) \beta+1)^{3} \frac{d^{2} \Theta_{1}}{d \tau^{2}}=0, \\
& \delta \theta_{2}:-\frac{1}{2} Q_{44} \mu((\zeta) \beta+1) \frac{d W}{d \eta} \\
& +\frac{1}{24} Q_{21} \alpha \mu((\zeta) \beta+1)^{3} \frac{d^{2} \Theta_{1}}{d \eta d \zeta} \\
& +\frac{1}{8} Q_{66} \alpha \mu((\zeta) \beta+1)^{2} \beta \frac{d \Theta_{1}}{d \eta} \\
& +\frac{1}{24} Q_{12} \alpha \mu((\zeta) \beta+1)^{3} \frac{d^{2} \Theta_{1}}{d \eta d \zeta} \\
& +\frac{1}{24} Q_{66} \alpha \mu((\zeta) \beta+1)^{3} \frac{d^{2} \Theta_{1}}{d \eta d \zeta} \\
& +\frac{1}{8} Q_{66} \alpha^{2}((\zeta) \beta+1)^{2} \beta \frac{d \Theta_{2}}{d \zeta} \\
& +\frac{1}{24} Q_{66} \alpha^{2}((\zeta) \beta+1)^{3} \frac{d^{2} \Theta_{2}}{d \zeta^{2}} \\
& +\frac{1}{12} Q_{22} \mu^{2}((\zeta) \beta+1)^{3} \frac{d^{2} \Theta_{2}}{d \eta^{2}} \\
& -\frac{1}{2} Q_{44}((\zeta) \beta+1) \Theta_{2}-\frac{1}{12} \alpha^{2}((\zeta) \beta+1)^{3} \frac{d^{2} \Theta_{2}}{d \tau^{2}} \\
& =0 \text {. }
\end{aligned}
$$

\section{Solution procedure using numerical method}

The DQM is a numerical technique for solving differential equations. As compared to the conventional low- order finite difference and FEMs, the DQM can obtain very accurate numerical results using a considerably smaller number of grid points and hence requiring relatively little computational effort.

\section{1. $1 D$ (Dimension) DQM}

Before utilizing DQM, the Navier solution is used considering the following form:

$$
\begin{aligned}
& U(\zeta, \eta, \tau)=U(\zeta) \sin (m \pi \eta) e^{\omega \tau}, \\
& V(\zeta, \eta, \tau)=V(\zeta) \cos (m \pi \eta) e^{\omega \tau}, \\
& W(\zeta, \eta, \tau)=W(\zeta) \sin (m \pi \eta) e^{\omega \tau}, \\
& \Theta_{1}(\zeta, \eta, \tau)=\Theta_{1}(\zeta) \sin (m \pi \eta) e^{\omega \tau}, \\
& \Theta_{2}(\zeta, \eta, \tau)=\Theta_{2}(\zeta) \cos (m \pi \eta) e^{\omega \tau},
\end{aligned}
$$

in which $\omega$ and $m$ are the dimensionless frequency and integer number, which are introduced as wave numbers. Navier solution converts the 2D equations of motion to a one-dimensional equation in which two edges of the plate are simply supported. Then, DQM is used where $F$ is a function representing $u, v, w, \theta_{1}$, and $\theta_{2}$ with respect to variable $\xi$ in the domain of $(0<\xi<L)$ [39]:

$$
\frac{\partial^{k} F}{\partial \zeta^{k}}=\sum_{k=1}^{N} C_{p q}^{(K)} F\left(\zeta_{i}\right)
$$

where $C_{p q}^{(k)}$ is the weighting coefficients associated with the $k$ th-order partial derivative of $F$, and $N$ is the number of grid points in longitudinal direction. Chebyshev polynomials [39] are selected for positions of the grid points. Substituting Eq. (17) into equations of motion, the standard form of vibrational equation $(M \ddot{X}+C \dot{X}+$ $K X=0)$ is derived. Considering the simply supported boundary condition that was explained in Mantari and Soares [40], the eigenvalues of state-space ma$\operatorname{trix}\left([\right.$ state - space $\left.]=\left[\begin{array}{cc}{[0]} & {[I]} \\ -[M K] & -[M C]\end{array}\right]\right)$ as dimensionless frequency are obtained. It is worth to mention that $M$ is the mass matrix, $C$ is the damping matrix, and $K$ is the stiffness; $[I]$ and $[0]$ are the unitary and zero matrices.

\subsection{The $\delta$-technique}

The $\delta$-technique was proposed to eliminate the difficulties in implementing two conditions at a single boundary point. In $\delta$-technique, Dirichlet condition $(W=0)$ is applied at the boundary point and the derivative condition at its adjacent point which is at a distance $\delta$ form the boundary point. As shown in Figure 2(a), the $\delta$-points are actually the grid points 
Table 1. Elastic properties of Terfenol-D [27].

\begin{tabular}{ccccc}
\hline Properties & $E$ & $v$ & $\rho_{m}$ & $e_{31}=e_{32}$ \\
\hline Terfenol-D & $30 \times 10^{9} \mathrm{~Pa}$ & 0.25 & $9.25 \times 10^{3} \mathrm{~kg} / \mathrm{m}^{3}$ & $442.55 \mathrm{~N} /(\mathrm{m} . \mathrm{A})$ \\
\hline
\end{tabular}

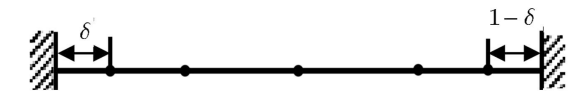

(a)

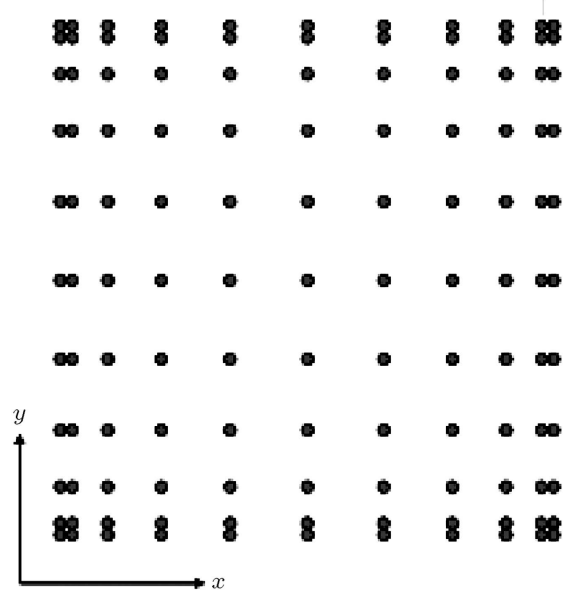

(b)

Figure 2. Positions of the grid points in (a) $\delta$-technique [39], and (b) 2D method.

$X_{2}=\delta$ and $X_{N-1}=1-\delta$. As an example for simply supported condition, $\frac{\partial^{2} W}{\partial X^{2}}=0$ is written as:

$$
\sum_{k=1}^{N} C_{2, k}^{(2)} \cdot W_{k}=0, \quad \text { and } \quad \sum_{k=1}^{N} C_{N-1, k}^{(2)} \cdot W_{k}=0 .
$$

\subsection{D $D Q M$}

In this case, there is no need to apply Eq. (17) and the problem is solved in $2 \mathrm{D}$ space as shown in Figure $2(\mathrm{~b})$. Firstly, the $x$ and $y$ derivations are introduced using weighting coefficients as follows:

$$
\begin{aligned}
& \left.\frac{\partial^{r} F}{\partial \zeta^{r}}\right|_{(x, y)=\left(x_{i}, y_{j}\right)}=\sum_{n=1}^{N} C_{i n}^{(r)} F_{n j}, \\
& \left.\frac{\partial^{r} F}{\partial \eta^{r}}\right|_{(x, y)=\left(x_{i}, y_{j}\right)}=\sum_{m=1}^{M} C_{j m}^{(r)} F_{i m}, \\
& \left.\frac{\partial^{r+s} F}{\partial \zeta^{r} \partial \eta^{s}}\right|_{(x, y)=\left(x_{i}, y_{j}\right)}=\sum_{n=1}^{N} \sum_{m=1}^{M} C_{i n}^{(r)} C_{j m}^{(s)} F_{n m}, \\
& i=1,2, \cdots, N, \quad j=1,2, \cdots, M, \\
& r=1,2, \cdots, N-1, \quad s=1,2, \cdots, M-1 .
\end{aligned}
$$

Then, the standard form of motion equation is solved like that in $1 \mathrm{D}$ method using eigenvalues problem. It is worth to mention that Chebyshev polynomials [39] are also selected for positions of the grid points.

\section{Numerical results and discussion}

In this work, motion equations of tapered $\mathrm{MsP}$ using FSDT were obtained. To account for the inaccuracy in the shear strain, a shear correction factor was applied so that the correct amount of internal energy was predicted by the theory. Also, a feedback system was used to control the vibration of $\mathrm{MsP}_{\mathrm{s}}$ in presence of magnetic field. Then, the vibration response of $\mathrm{MsP}$ was investigated by stimulus factors such as velocity feedback gain, boundary condition, aspect ratio, thickness ration, and taper ratio.

The plate is made of Terfenol-D and its properties are listed in Table 1.

In order to approve the accuracy of the solution method, results of this work were compared with the computed results of those available in the literature for the isotropic case (Eq. (16) from Refs. [12,41]). Table 2 reports the results of seven different solution methods. As can be seen, Table 2 shows the good accuracy of the obtained results.

Figure 3 shows the variation of dimensionless frequency versus thickness ratio of $\mathrm{MsP}$ in different taper ratios. $\alpha$ changes from 0.1 to 0.3 for thick plates and as can be seen in the figure, increase in aspect ratio leads to increase in frequency due to the effect of mass and inertia. Also, by increasing the slope of thickness function that is known as taper ratio, the frequency of $\mathrm{MsP}$ changes and increases, like what has been reported in $[10]$.

Figure 4 illustrates variation of dimensionless frequency versus thickness ratio of $\mathrm{MsP}$ in presence of magnetic field. It is worth to mention that when the MsMs are subjected to magnetic field, they deform due

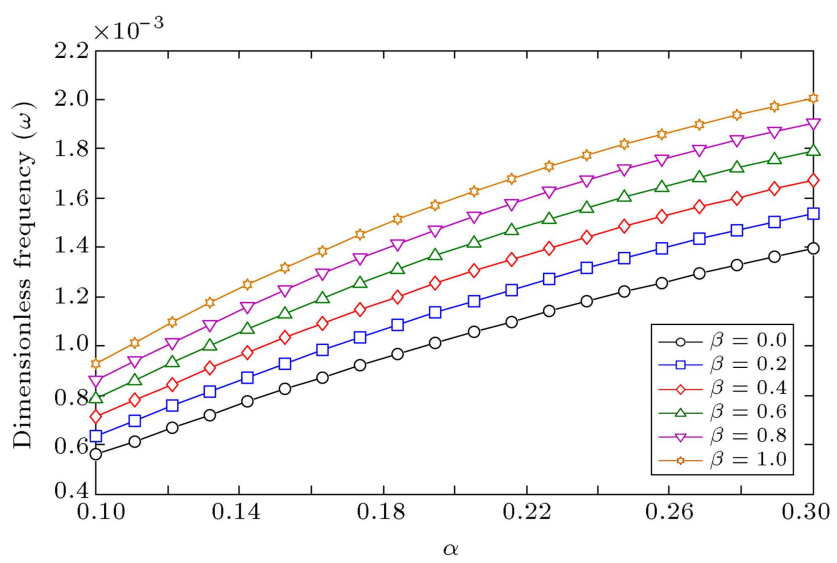

Figure 3. Variation of dimensionless frequency versus thickness ratio in different taper ratios $\left(\gamma=1, K_{c} C(t)=10^{8}\right)$. 
Table 2. The comparison of results for different solution methods.

\begin{tabular}{ccccccccc}
\hline $\boldsymbol{\gamma}$ & $\boldsymbol{\beta}$ & $\boldsymbol{\delta}$-technique & 2D & 1D & Ref. [12] & Ref. [12] & Ref. [12] & Ref. [41] \\
\hline & 0.1 & 20.7108 & 20.7105 & 20.7107 & 20.7296 & 20.7122 & 20.6237 & 20.7206 \\
& 0.2 & 21.6544 & 21.6541 & 21.6543 & 21.7025 & 21.6506 & 21.5905 & 21.6919 \\
& 0.3 & 22.5732 & 22.5729 & 22.5731 & 22.6669 & 22.5533 & 22.5486 & 22.6529 \\
\multirow{1}{1}{} & 0.4 & 23.4699 & 23.4696 & 23.4698 & 23.6239 & 23.4203 & 23.4987 & 23.6049 \\
& 0.5 & 24.3468 & 24.3465 & 24.3467 & 24.5740 & 24.2519 & 24.4418 & 24.5563 \\
& 0.6 & 25.2058 & 25.2055 & 25.2057 & 25.5175 & 25.0490 & 25.3784 & 25.4961 \\
& 0.7 & 26.0489 & 26.0485 & 26.0487 & 26.4547 & 25.81263 & 26.3092 & 26.4288 \\
& 0.8 & 26.8776 & 26.8773 & 26.8775 & 27.3845 & 26.5440 & 27.2346 & 27.3535 \\
\hline
\end{tabular}

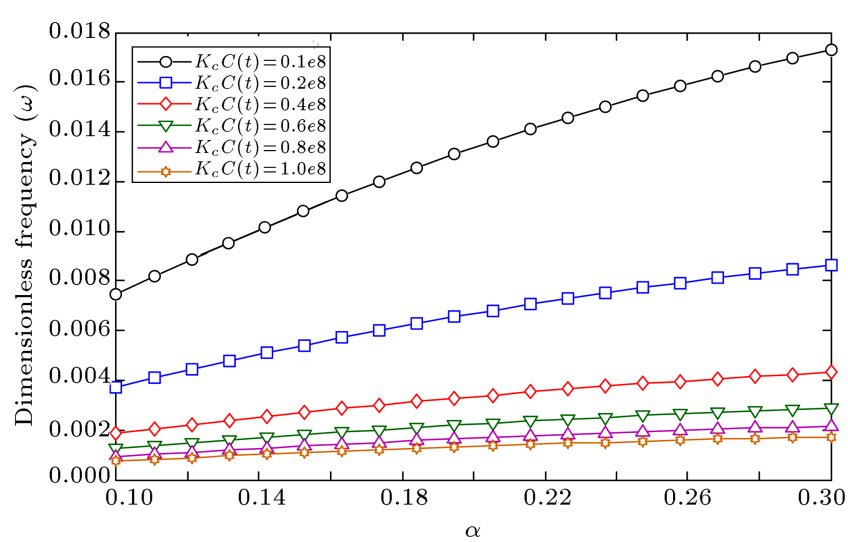

Figure 4. Variation of dimensionless frequency versus thickness ratio in different velocity feedback gains $(\gamma=1, \beta=0.5)$.

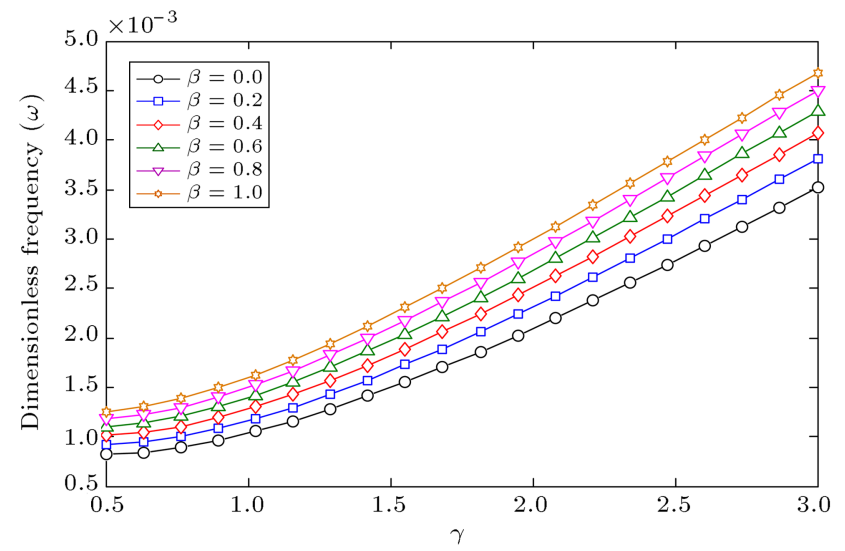

Figure 5. Variation of dimensionless frequency versus aspect ratio in different taper ratios $\left(\alpha=0.2, K_{c} C(t)=10^{8}\right)$.

to reciprocal nature. It is possible to control MsP frequency by change in velocity feedback gain parameter $K_{c} C(t)$. As can be seen in Figure 4, the frequency of $\mathrm{MsP}$ significantly decreases with increasing velocity feedback gain.

The changes of dimensionless frequency of tapered $\mathrm{MsP}$ versus aspect ratio in different taper ratios and velocity feedback gains are demonstrated in Figures 5 and 6 , respectively. It is clear in figures that increasing

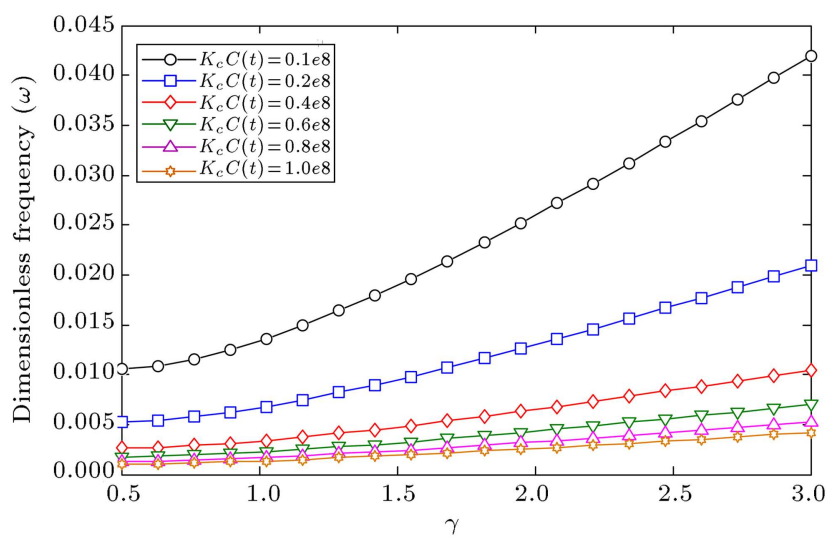

Figure 6. Variation of dimensionless frequency versus aspect ratio in different velocity feedback gains $(\alpha=0.2, \beta=0.5)$.

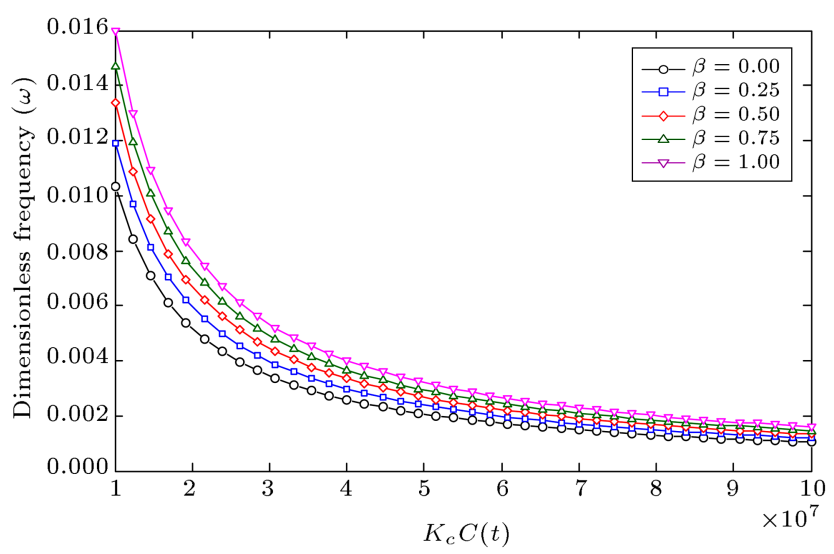

Figure 7. Variation of dimensionless frequency versus positive velocity feedback gains in different taper ratios $(\gamma=1, \alpha=0.2)$.

the aspect ratio from $\gamma=0.5$ to 3 leads to the development of frequency as it is concluded in [10]. Also, Figure 5 shows that increasing the thickness of $\mathrm{MsP}$ in $x$ direction increases the natural frequency of $\mathrm{MsP}$ while increase in velocity feedback gain has contrary effect.

Figures 7 and 8 clearly show the effect of magnetic field on tapered MsP by change in velocity feedback gain. These figures reveal how much effective the 


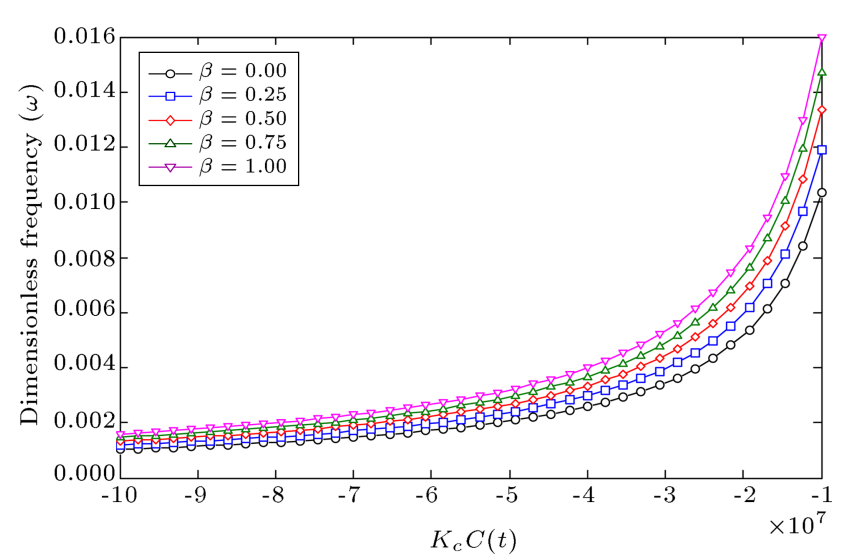

Figure 8. Variation of dimensionless frequency versus negative velocity feedback gain in different taper ratios $(\gamma=1, \alpha=0.2)$.

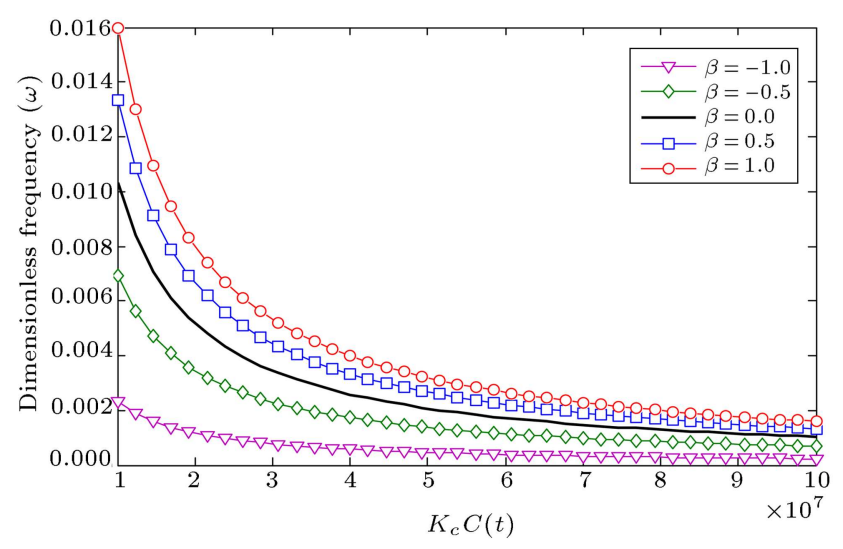

Figure 9. The comparison of results in positive and negative taper ratios $(\gamma=1, \alpha=0.2)$.

magnetic field intensity is on natural frequency of tapered $\mathrm{MsP}$. Increase in velocity feedback gain from $K_{c} C(t)=10^{7}$ to $10^{8}$ significantly decreases the natural frequency of tapered MsP, as shown in Figure 7. Also, the reverse magnetic field can increase the natural frequency of tapered $\mathrm{MsP}$ with the same intensity. Results show that the vibration of tapered MsP can be controlled by velocity feedback gain parameter.

Figure 9 has been plotted to display the effect of positive and negative values of taper ratio. It shows the effect of magnetic field on tapered $\mathrm{MsP}$ by change in velocity feedback gain. As can be seen in Figure 9, the negative taper ratios create lower frequency considering the dimension of MsP. The similar results have been reported in [15].

Figure 10(b) has been drawn to show the effect of different boundary conditions that are displayed in Figure 10(a) on natural frequency of taper $\mathrm{MsP}$. Since the governing equations have been solved by $1 \mathrm{D}$ DQM, two opposite edges are simply supported that are numbered by 1 and 2 in Figure 10(a). Changing the boundary condition of edges by numbers 3 and 4 leads to variation in natural frequency, especially at lower

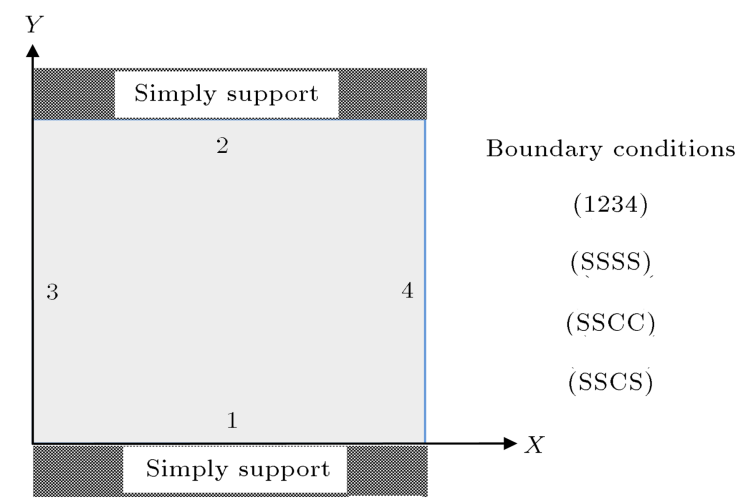

(a)

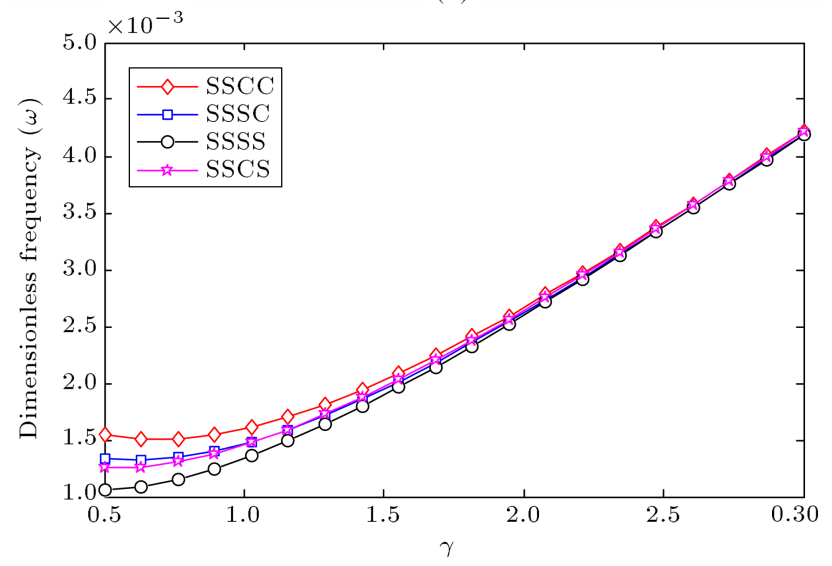

(b)

Figure 10. (a) Display of different boundary conditions on four edges of tapered MsP. (b) Effect of different boundary conditions on dimensionless frequency of tapered $\operatorname{MsP}\left(\alpha=0.2, \beta=0.5, K_{c} C(t)=10^{8}\right)$.

thickness ratios. Also, with increasing $\gamma$, the natural frequency for different boundary conditions converge to the same value. The comparison among curves designates the stable cases, respectively, in $\gamma=1$, $\alpha=0.2, \beta=0.5$, and $K_{c} C(t)=10^{8}$ as follows:

$$
\mathrm{SSCC}>\mathrm{SSSC}>\mathrm{SSCS}>\mathrm{SSSS} \text {. }
$$

Figure 11(a) and (b) show the variation of dimensionless frequency versus different wave numbers. All of the figures in the present work were plotted for $m=1$. As can be seen in Figure 10(b), the differences are clearer in high wave numbers. According to Figure 11(a), which is $3 \mathrm{D}$ the $\mathrm{MsP}$ is larger (with high $\gamma$ and $\alpha$ ) and the variation is more obvious.

All of the figures in the present work (Figures 3 to 11 ) were plotted by considering the shear correction factor. Since $v=0.25$ for Terfenol-D, the shear correction factor is calculated $k_{f}=0.86$ by Timoshenko relation, which is much close to $k_{f}=5 / 6=0.83$, and the difference between the results is very negligible. Figure 12 approves that in $\mathrm{MsP}$ with large dimensions, the shear correction factor must be considered; otherwise, the results are not accurate. 


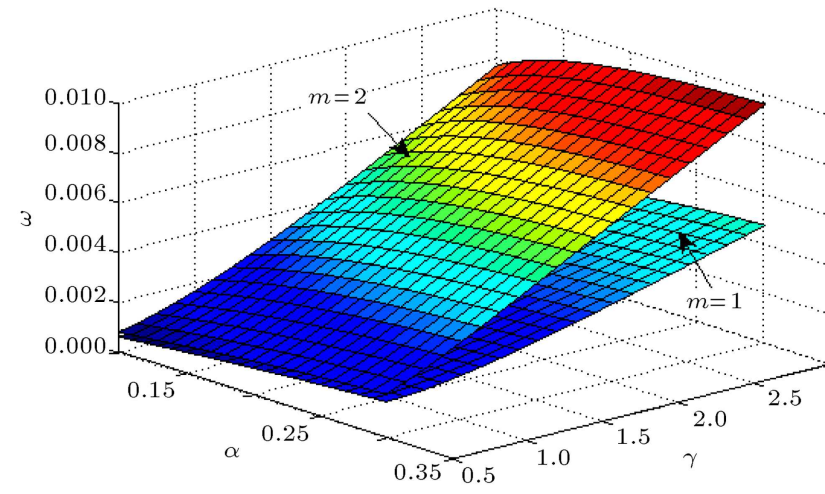

(a)

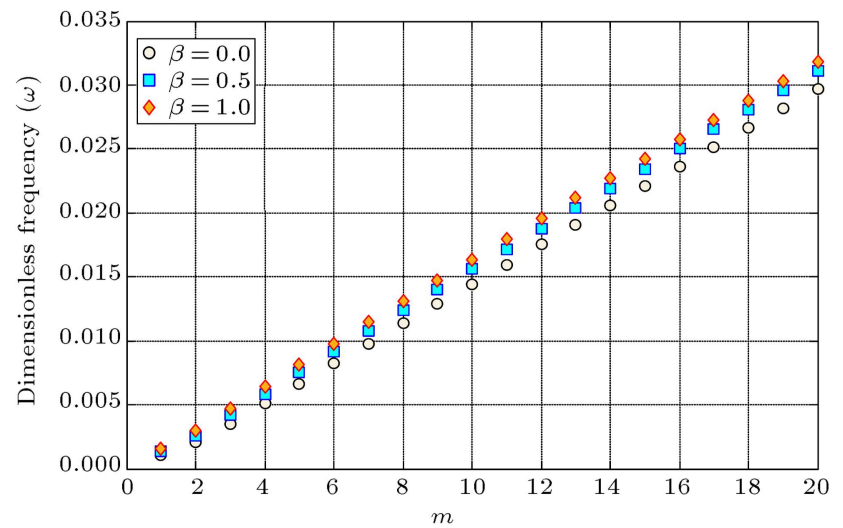

(b)

Figure 11. (a) Variation of dimensionless frequency versus thickness ratio and aspect ratio at two different wave numbers $\left(\beta=0.5, K_{c} C(t)=10^{8}\right)$. (b) Variation of dimensionless frequency of tapered MsP versus different wave numbers $\left(\alpha=0.2, \gamma=1, K_{c} C(t)=10^{8}\right)$.

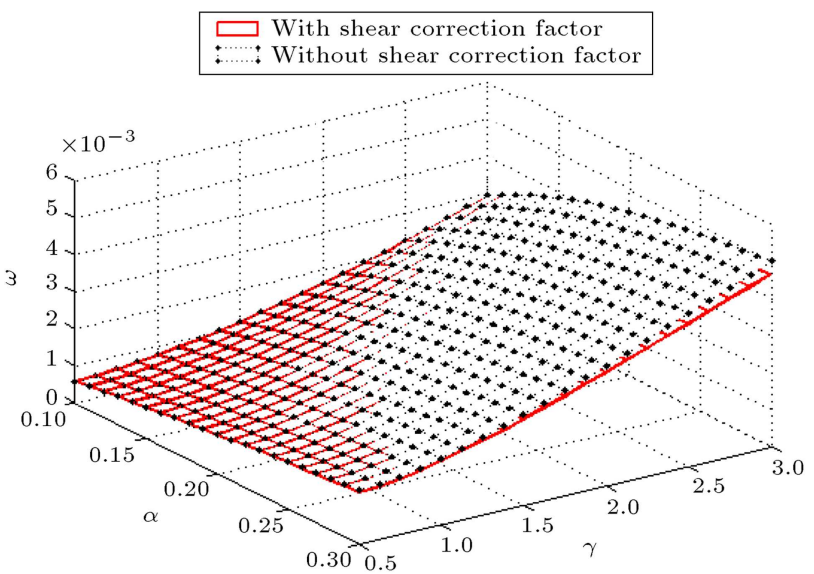

Figure 12. Effect of shear correction factor on dimensionless frequency of tapered MsP $\left(\beta=0.5, K_{c} C(t)=10^{8}\right)$.

\section{Conclusion}

At the first time, free vibration of tapered plate made of MsM was evaluated using feedback control system. Thickness of $\mathrm{MsP}$ varied linearly in $x$ direction by $h(x)=h_{0}(1+\beta x / a)$. Considering shear correction factor in FSDT, equations of motion were derived and solved by numerical method for different boundary conditions. Results showed the effect of main parameters on frequency response of $\mathrm{MsP}$ that is presented below:

- Increasing the thickness ratio in the interval $0.1 \leq$ $\alpha \leq 0.3$ for thick plate increases the dimensionless frequency of MsP;

- Increasing the aspect ratio $(0.5 \leq \gamma \leq 3)$ increases the dimensionless frequency of $\mathrm{MsP}$;

- Increasing the taper ratio $(0 \leq \beta \leq 1)$ increases the dimensionless frequency of $\mathrm{MsP}$;

- Disregarding shear correction factor causes inaccuracy in the results at the large dimensions;

- Increasing the velocity feedback gain as a control parameter can reduce the frequency of $\mathrm{MsP}$ and makes the other parameters $(\alpha, \gamma, \beta)$ become ineffective.

According to the above results, $\mathrm{MsP}$ can be used to designate noise, shock, resonance, and control of vibrational response of systems.

\section{Acknowledgments}

The authors would like to thank the reviewers for their comments and suggestions to improve the clarity of this article. This work was supported by University of Kashan [grant number 574600/5].

\section{References}

1. Wang, L. "Vibration energy harvesting by magnetostrictive material for powering wireless sensors", A Thesis Submitted to the Graduate Faculty of North Carolina State University, Raleigh, NC (2007).

2. Liu, J.P., Fullerton, E., Gutfleisch, O. and Sellmyer, D.J., Nanoscale Magnetic Materials and Applications, Springer Publisher, New York (2009).

3. http://www.mtssensors.com/dev-pages/orphanpages/technology/how-magnetostriction-works/ index html.

4. Moon, F.C. and Pao, Y.H. "Magnetoelastic buckling if a thin plate", J. Appl. Mech., 35, p. 53 (1986).

5. Wang, X. and Lee, J.S. "Dynamic stability of ferromagnetic plate under transverse magnetic field and inplane periodic compression", Int. J. Mech. Sci., 48, pp. 889-898 (2006).

6. Moita, J.M.S., Soares, C.M.M. and Soares, C.A.M. "Analyses of magneto-electro-elastic plates using a higher order finite element model", Compos. Struct., 91, pp. 421-426 (2009).

7. Hosseini Hashemi, S.H., Rokni Damavandi Taher, H., Akhavan, H. and Omidi, M. "Free vibration of functionally graded rectangular plates using first-order shear deformation plate theory", Appl. Math. Model., 34(5), pp. 1276-1291 (2010). 
8. Hong, C.C. "Thermal sinusoidal vibration and transient response of magnetostrictive functionally graded material plates without shear effects", Researches and Applications in Mechanical Engineering, 2, pp. 11-22 (2013).

9. Hong, C.C. "Thermal vibration and transient response of magnetostrictive functionally graded material plates", Eur. J. Mech. A. Solids., 43, pp. 78-88 (2014).

10. Civalek, O. "Fundamental frequency of isotropic and orthotropic rectangular plates with linearly varying thickness by discrete singular convolution method", Appl. Math. Modell., 33, pp. 3825-3835 (2009).

11. Huang, M., Ma, X.Q., Sakiyama, T., Matuda, S. and Morita, C. "Free vibration analysis of orthotropic rectangular plates with variable thickness and general boundary conditions", J. Sound Vib., 288, pp. 931-955 (2005).

12. Kukeriti, A.R., Farsa, J. and Bert, C.W. "Differential quadrature and Rayleigh-Ritz methods to determine the fundamental frequencies of simply supported rectangular plates with linearly varying thickness", $J$. Sound Vib., 189, pp. 103-122 (1996).

13. Gupta, A.K. and Sharma, S. "Effect of thermal gradient on vibration of non-homogeneous orthotropic trapezoidal plate of linearly varying thickness", Ain Shams Eng. J., 4, pp. 523-530 (2013).

14. Kang, S.W. and Kim, S.H. "Vibration analysis of simply supported rectangular plates with unidirectionally, arbitrarily varying thickness", J. Sound Vib., 312, pp. 551-562 (2008).

15. Singh, B. and Saxena, V. "Transverse vibration of a rectangular plate with bidirectional thickness variation", J. Sound Vib., 198, pp. 51-65 (1996).

16. Gupta, A.K. and Khanna, A. "Vibration of viscoelastic rectangular plate with linearly thickness variations in both directions", J. Sound Vib., 301, pp. 450-457 (2007).

17. Tounsi, A., Houari, M.S.A., Benyoucef, S. and Adda Bedia, E.A. "A refined trigonometric shear deformation theory for thermoelastic bending of functionally graded sandwich plates", Aerosp. Sci. Technol., 24, pp. 209-220 (2013).

18. Belabed, Z., Houari, M.S.A., Tounsi, A., Mahmoud, S.R. and Anwar Bég, O. "An efficient and simple higher order shear and normal deformation theory for functionally graded material (FGM) plates", Composites: Part B, 60, pp. 274-283 (2014).

19. Hebali, H., Tounsi, A., Houari, M.S.A., Bessaim, A. and Adda Bedia, E.A. "A new quasi-3D hyperbolic shear deformation theory for the static and free vibration analysis of functionally graded plates", ASCE J. Engineering Mechanics, 140, pp. 374-383 (2014).

20. Zidi, M., Tounsi, A., Houari, M.S.A., Adda Bedia, E.A. and Anwar Bég, O. "Bending analysis of FGM plates under hygro-thermo-mechanical loading using a four variable refined plate theory", Aerosp. Sci. Technol., 34, pp. 24-34 (2014).
21. Draiche, K., Tounsi, A. and Khalfi, Y. "A trigonometric four variable plate theory for free vibration of rectangular composite plates with patch mass", Steel and Composite Structures, 17(1), pp. 69-81 (2014).

22. Houari, M.S.A., Tounsi, A. and Anwar Bég, O. "Thermoelastic bending analysis of functionally graded sandwich plates using a new higher order shear and normal deformation theory", Int. J. Mech. Sci., 76, pp. 102111 (2013).

23. Bachir Bouiadjra, R., Adda Bedia, E.A. and Tounsi, A. "Nonlinear thermal buckling behavior of functionally graded plates using an efficient sinusoidal shear deformation theory", Structural Engineering and Mechanics, 48, pp. 547-567 (2013).

24. Bessaim, A., Houari, M.S.A., Tounsi, A. Mahmoud, S.R. and Adda Bedia, E.A. "A new higher-order shear and normal deformation theory for the static and free vibration analysis of sandwich plates with functionally graded isotropic face sheets", J. Sandwich Struct. Mater., 15(6), pp. 671-703 (2013).

25. Eftekhari, S.A. and Jafari, A.A. "Accurate variational approach for free vibration of variable thickness thin and thick plates with edges elastically restrained against translation and rotation", Int. J. Mech. Sci., 68, pp. 35-46 (2013).

26. Shufrin, I. and Eisenberger, M. "Vibration of shear deformable plates with variable thickness first-order and higher-order analyses", J. Sound Vib., 290, pp. 465-489 (2006).

27. Hong, C.C. "Transient responses of magnetostrictive plates without shear effects", J. Sound Vib., 47, pp. 355-362 (2009).

28. Hong, C.C. "Transient responses of magnetostrictive plates by using the GDQ method", Eur. J. Mech. A. Solids., 29, pp. 1015-1021 (2010).

29. Timoshenko, S.P. "On the transverse vibrations of bars of uniform cross-section", Philos. Mag. A., 43, pp. 125131 (1922).

30. Jalili, N., Piezoelectric-Based Vibration Control from Macro to Micro/Nano Scale Systems, Springer Science, Boston (2010).

31. Chen, J.Y., Heyliger, P.R. and Pan, E. "Free vibration of three-dimensional multilayered magnetoelectro-elastic plates under combined clamped/free boundary conditions", J. Sound Vib., 333, pp. 40174029 (2006).

32. Pan, E. and Heyliger, P.R. "Free vibration of simply supported and multilayered magneto electro plates", J. Sound Vib., 252, pp. 429-442 (2002).

33. Krishna, M., Anjanappa, M. and Wu, Y.F. "The use of magnetostrictive particle actuators for vibration attenuation of flexible beams", J. Sound Vib., 206, pp. 133-149 (1997).

34. Lee, S.J., Reddy, J.N. and Rostamabadi, F. "Transient analysis of laminated composite plates with embedded 
smart-material layers", Finite Elem. Anal. Des., 40, pp. 463-483 (2004).

35. Reddy, J.N., Energy Principles and Variational Methods in Applied Mechanics, John Wiley \& Sons Publishers, Texas (2004).

36. Mindlin, R.D. "Influence of rotatory inertia and shear on flexural motions of isotropic, elastic plates", $A S M E$ Journal of Applied Mechanics, 18, pp. 31-38 (1951).

37. Rastgaar Aagaah, M., Nakhaie Jazar, G. and Nazari, G. "Third order shear deformation theory for modeling of laminated composite plates", SEM X International Congress and Exposition on Experimental and Applied Mechanics, Costa Mesa, CA (June 7-10, 2004).

38. Thai, H.T. and Choi, D.H. "Improved refined plate theory accounting for effect of thickness stretching in functionally graded plates", Composites: Part B., 56, pp. 705-716 (2014).

39. Shu, C., Differential Quadrature and Its Application in Engineering, Springer publishers, Singapore (2000).

40. Mantari, J.L. and Soares, C.G. "A novel higher-order shear deformation theory with stretching effect for functionally graded plates", Composites: Part B., 45, pp. 268-281 (2013).

41. Appl, F.C. and Byers, N.R. "Fundamental frequency supported rectangular plate with linearly varying thickness", J. Mech. Des-T Asme., 32, pp. 163-168 (1965).

\section{Biographies}

Ali Ghorbanpour Arani received his BSc degree from Sharif University of Technology in Tehran, Iran, in 1988. He then received his MSc degree from Amirkabir University of Technology in Tehran, Iran, in 1991, and his PhD degree from Esfahan University of Technology in Esfahan, Iran, in 2001. Dr. Ali Ghorbanpour Arani is currently a Professor in the Mechanical Engineering Faculty at University of Kashan in Kashan, Iran. He has authored more than 200 refereed journal papers and 10 books. His current research interests are stress analyses, stability and vibration of nanostructures, NEMs, MEMs, and FGMs.

Zahra Khoddami Maraghi received her BSc degree in 2008 and then the MSc degree in 2011 from University of Kashan in Kashan, Iran. She is currently a PhD student at University of Kashan. She has authored more than 11 refereed journal papers and 1 book. Her research interests are control, vibration, and instability of nanostructures and smart materials.

Hamed Khani Arani received his BSc degree from University of Kashan in Kashan, Iran, in 2012. He is currently an MSc student at University of Kashan. His research interests are vibration of smart-structure and nanomechanics. 